\title{
Technology, new business models, and the future of the fashion brand
}

\begin{abstract}
The need for branding, as we know it in modern times, started as a result of the industrial revolution. Then in the second half of the twentieth century, the technological advancements in communication and marketing tools together with flourishing economies and increase in consumerism made branding the buzzword of marketing strategies. However, in recent years new technological advancements such as the rise of the internet and further advances in communication have resulted in the creation of new tools and business models that are directly impacting old school marketing practices. Examples of such models are mass customization and open innovation, both of which have created a new framework that is having a strong impact on the traditional product development cycle and the way marketers need to approach and define the "brand". For example, a new paradigm established by such models has repositioned the consumer in the middle of the brand development process as one of the co-authors and not just a mere end user. As a result, and instead of the traditional "one brandmulti products" framework, we are starting to witness a multitude of variations of the same brand (and not just the product) and the creation of a series of "micro" or "satellite" brands". The formation of such micro-brands forces us to reconsider the way we should define and approach the brand concept in the twenty first century. This paper aims at exploring and explaining the reasons behind the creation of "microbrands" as well as attempts to suggest a new definition of the brand in a manner that would reflect the new paradigm.
\end{abstract}

Keywords: branding, innovation, technology, marketing, micro branding, mass customization, open innovation
Volume 2 Issue I - 2017

Kaled K Hameide

Montclair State University, USA

Correspondence:Kaled K Hameide, Department of Art and Design, Montclair State University, USA, Tel 9736554I79, Email hameidek@mail.montclair.edu

Received: March 24, 2017 | Published: June 14, 2017

\section{Introduction}

Although the industrial revolution could be credited for the birth of branding in modern times, it wasn't until the second half of the twentieth century that branding as we know it today exploded in the business scene. Technological advancements in communication and marketing tools together with flourishing economies and increase in consumerism made branding the buzzword of the eighties. Since then, everything seemed prone to become branded. By the end of the twentieth and the beginning of the twenty first centuries we have witnessed a communication revolution with the birth of the internet and other technological advances in mobile and wireless technologies. From a marketing perspective these developments had an equally dramatic impact, creating a range of new products and services and in return new marketing tools and strategic opportunities. As with any developments of such nature and magnitude, new business models develop to respond to these needs and benefit from these opportunities. Mass Customization and Open Innovation were two models that have utilized such new platforms and proved to have an impact on the traditional product development process. While the impact of such models has been thoroughly examined from the logistic, production, and inventory management perspectives, their impact on marketing strategies and most importantly on the core of such strategies, which is the brand, has not been equally tackled. In this paper we will attempt to focus on that important perspective and examine the impact of these innovations on the future of the brand and the branding process.

\section{The brand logic}

Brands exist mainly to reduce risks for consumers by encouraging them to buy a branded product that they can trust and anticipate its level of quality and service. Thus, brands make it easier for consumers to make safer choices that will eventually satisfy their needs as promised by the brand and in the process provide a certain reward or "value" that can be either emotional or rational.

Brands are also built on the premises of differentiation and bringing something new and different that other competitors in the market cannot, i.e. a new idea or concept packed with a mix of offerings and features that are different and unique. The more unique and distinctive the idea, the more legitimate and valid the brand will be, and the more attractive as well.

Once a brand is recognized and appreciated by the customers, it aims at keeping them attached and loyal by consistently living up to their expectations and remaining relevant and fresh.

\section{The definition}

Based on the above stated logic behind having a "brand," we could put together a definition that captures that rationale as well as the essence of the term from a holistic perspective:

A. A brand is an entity with a distinctive idea expressed in a set of functional and experiential features with a promise of a value reward relevant to its end user, and an economic return to its producers (through the building of equity). A successful brand has a strong identity (mentally and physically), is innovative, consistent, competitively positioned, and holds a matching positive image in the consumer's mind.

B. This definition captures the essence and role of the "brand" as we understand and perceive it today. It highlights the produc- 
ts (or service) as the core of the brand and acknowledges the other elements that complement the functional attributes of its physical product. It also implies that branding is indeed a story, a process, and that a brand is in many ways a living being with a life cycle that pretty much resembles that of ours (i.e. birth-growth-decline-demise). It is built on features and meanings, on functions and emotions, as well as on history and future prospects. This is important, as we will need to revisit the definition as we examine the impact of new technological and business models on the role of the brand.

\section{Who is responsible for the brand?}

If a brand is a story, then who is responsible for creating it? A holistic perspective will show that the brand story is actually cowritten by three main partners: The Company, the consumer, and the culture (and external environment).

Culture: Culture dictates new social trends, beliefs and behaviors. It defines what is "cool" and acceptable by various age groups and market segments.

Company: The Company is the producer of the brand and the one that bares the ultimate financial and marketing risks as well as the creative, production, and logistic responsibilities of developing the brand.

Customer: Brand customers experience the brand and consume its products and therefore play an important role in the life of the brand. For starters, a brand is developed with the end users in mind and with the goal of satisfying their unfulfilled needs. In addition, the users are the ultimate judges of the brand, and it is they who decide the brand's true and final positioning based on their experiences with it, among other factors. Accordingly, we could conclude the following simple yet important equation:

\section{The Culture + The Company + The Consumer $=$ The Brand}

The equation is important because it highlights the relevance of external factors in developing the brand. However, while this equation acknowledges the importance of the consumer in the branding process, classic marketing perspective implies a rather passive role for him/her by placing them merely at both ends of the branding process. Seen as the inspiration or motivator at one side and the end user at another, he/she is clearly missing from the development process except for the occasional input that is selectively administrated by companies through market research and consumer feedback channels. However, this relationship seems bound to change based on the new market realities as will be made clearer as we proceed with our discussion.

\section{Technology and new business models}

Technological and communication innovations such as the internet, mobile and wireless technologies have helped introduce new tools, capabilities and business models that among many things enabled the consumer to directly interact with the brand and play a rather direct role in its development and marketing. By examining each and every one of these innovations we will notice how consumer “empowerment' consistently occurs as the major outcome. It's a development that as we will discover redefines the role of players and reposition them in the brand development process. A good example of these new trends and models is the concept of mass customization.

\section{Mass customization}

Mass Customization (MC) refers to the concept of allowing each consumer to customize or adapt products' features according to his/ her needs within a standardized platform and with an acceptable price premium.

According to the above definition, mass customization could be seen as middle of the road between mass production and pure customization as it gives consumers an option to customize features of a product, given that it is based on a mass-produced structure or frame. There are many successful examples of MC from various industries such as:

i. Threadless.com, in Apparel: probably one of the most successful examples offering mass customized $t$-shirts online.

ii. Dell, in Computers: mass customized computers and laptops.

iii. Mi Adidas from Adidas, In Sports Gear: mass customized sport shoes for the Adidas brand.

iv. PersonalNovel.de, in Publishing: an online company that offers customers the opportunity to customize novels.

On the other hand, a similar model known as Open Innovation (OI) refers to an environment of community sourcing, whereby every new input element might add to or tweak an existing distinctive feature such as the Linux operating system.

In each one of the above examples, the consumer plays a direct role in designing the final product that he would to consume which in itself demonstrates an interesting and significant paradigm shift in the way and level by which companies manage their brands and interact with the final user. In addition, the model promises to create a product that brings a high level of satisfaction as the product is co-designed by its end user according to their specific needs and desires. However, it does raise a couple of other intriguing questions such as: How do these developments and technologies while affecting the nature of the product would in return affect the brand concept? And would the above stated "classic" perspective and definition for the brand still holds or do we need to develop a new definition as we re-examine the branding process?

To approach these questions and the implications of such developments we will start by examining the following hypothetical scenario.

\section{The coca cola dilemma}

Some soda drinkers who would like to switch to a diet soda drink such as Diet Coke for example, are held back by the difference in taste of the sugar-free drink. Let us assume that with the help of technological innovation a company like Coca Cola manages to develop a vending machine that allows customers to adjust the sweetness level of the coke to their taste so that each customer will still be getting a low calorie diet drink yet with an acceptable taste. While that would be a great application of mass customization it would raise a series of questions such as: How would we define the diet coke brand now? And what does diet coke mean for each of its consumers? We will attempt to answer these questions as we proceed.

We already understand that a brand has in its core a product with one or more distinctive functional features that posses a strong identity and delivers a value to the user. We have also seen that mass customization (and open innovation) allows each user to alter the product by manipulating and altering its features (or adding to it). However, we can easily deduce that manipulating and altering 
products' features does not in itself alter the brand, otherwise every product update would mean creating a new brand. Yet it does impact the brand in the form of creating new brand extensions or rather a series of what we may call "micro-brands". "Micro-brands" could be seen as a group of satellite brands that are variations of the mother brand.

\section{Micro-brands}

By definition, a mass-customized brand (MC brand) is a brand where every consumer decides on the distinctive feature of his/her customized item. In both cases, the end result is a variety of distinctive features and variations of the "mother brand." This variety of features ends up creating what we may call a series of "Micro" (or satellite) brands, where a micro brand is a limited yet distinct version of the original (or mother) one.

It is essential to remember that mass customization is a middle of the road option between cookie-cutter mass production and total bespoke made-to measure, or one-of-a-kind products. There is still a level of commonality and control, which makes it necessary to link these brands to the mother brand and create a common base and identity that links these satellite brands together. It is a confirmation that they are still the same family of siblings but simply not the twins they used to be. This is why mass customization is usually done at the product level but not at the brand identity level, which needs to remain intact as the umbrella or back bone that defines the mother brand and maintains the brand personality and ultimately its core value.

Accordingly, we can say that: Satellite micro-brands are a set of sub brands with a common unifying identity and feature base, yet with a score of distinctive features customized and defined by the end user; these customized features create a unique experience with an equally high level of satisfaction among users and ultimately a higher level of loyalty to the mother brand. Micro-brands are obviously harder to predict since they are actually created and shaped by the user. In addition, they impose some logistic and production challenges as the platform needs to be flexible enough to accommodate the unplanned variations and the limitations in quantity. On the other hand, the level of control, such as the common platform" imposed by the producer, minimizes some of these challenges.

\section{Micro-branding vs. brand extensions}

We have described micro brands as a form of brand extensions, yet the difference between brand extensions and micro brands is that brand extensions are about creating new and totally different products under the same brand (e.g. a fashion brand extends to home furnishings under the same name) but here the new created products share a commonality of purpose (my customized Adidas running shoes as well as yours are still running shoes, your customized Levis jeans are still that and so is every mass customized product but each in its own way). They are simply variations in a theme. Thus the key issue is that these changes while they may alter the product features and enhance its functionality should not alter the brand meaning and value. If you and I can create our own Adidas running shoes we will get different shoes but not necessarily a different experience or brand value on the contrary we should have a closer level of experiential satisfaction. This is referred to as experiential branding.

\section{Micro-brands and experiential branding}

At the end of the day what branding really does is create an experience, i.e. while products are consumed, brands are actually experienced. Thus, we can conclude that the goal of any brand and its marketing strategies is to "deliver the elements that would trigger these experiences. The belief is that the same set of experience components delivered to a group of people who share similar sensory perceptions, emotional drivers, personal histories and living environments will trigger effectively similar experiences in each one of these people." This is referred to in marketing as "experiential segmentation" and "experiential branding." Experiential branding is "the discipline of understanding and defining brands in terms of the way they are experienced, in order to differentiate them in the most powerful dimension: relevance (nothing is more relevant than an experience)."

In reality, this seems hard to achieve, not just because each user is subject to different external factors (or noise) but because the products (which are traditionally a constant) are subject to different users' perceptions (a variable); thus, while you and I may be buying the same exact product, we most likely will have different experiences. However, if we refer again to our customized Adidas running shoes that are attainable because of new technologies, what we would have here is a situation where the product and its features are not identical anymore, and the product is no longer a constant. Yet, most likely, each of the users is getting an almost equal level of emotional satisfaction. Thus, while each user would define the branded product differently, it would satisfy all users in a closer way. Experiences are generally measured by the level of satisfaction, and in this case the users' experiences generate closer levels of satisfaction than could have been achieved before when the same product was meant to satisfy different people under different perceptions.

It is important to remember that while mass customization alters the product's features and thus functionality, these alterations caused to the features are never meant to be big enough to shift the product into a different category (i.e. running shoes fail to function as such and accordingly move into a different category); to achieve this, customization has to be handled with a certain level of control and limitations, which is exactly what mass customization is all about. Therefore, the emotional reward and satisfaction would outweigh the discrepancy in functionality and features and manage to generate an equally satisfying experience for all users.

So now we can conclude that mass customization does not necessarily create a new brand out of an old one but rather extend it into micro variations that may look and function slightly different yet deliver a better and rather consistent experience to all users.

\section{Micro-brands and positioning}

Positioning is an important concept in branding that refers to how the brand is perceived in the market by consumers relevant to its competitors. Thus it is important that we examine the impact of such changes on the concept. It is apparent that under the new environment, the few elements the company can fully control are the initial features, or the product base, along with the identity, tools, and options it allows the users to customize. Thus, the role of the company is really shifting more towards being a service provider or a facilitator. So what the customer/user is really looking for is a suitable and innovative environment that will make the experience and process simpler and more effective. Is it easy to manipulate the product? Does the platform or software offer necessary tools for manipulation? Does the system crash? Does the system offer a responsive and effective feedback mechanism? And so on. And just as the major focus of all service providers is the users' experience, positioning based on experience and the emotional value of the brand becomes essential and paramount. 
The classic concepts of product positioning and brand image may seem irrelevant for the most part since they are predetermined and established by the user before the product is even fully developed and consumed. Therefore, as a product, the brand is inherently positioned or pre-positioned by the ultimate user, which should be a marketer's dream come true, as the end product is predetermined, pre-designed, and pre-demanded by the end user. This might hypothetically mean that the producer is making a product that is pre-sold.

Thus, we can say that whenever the customer's intervention is high, the image tends to be more innovation-dependant (experiential positioning focused) while it is more features-driven (product positioning focused) when the customer's intervention is minimal. This will be clearly evident in the case of brands that possess some kind of a monopoly or "secret" formula, as in the case of pharmaceutical products or the case of Coke. These brands will be less susceptible to the customization of their monopoly feature, which in return will remain the core of the branding experience (and positioning strategy). The Coke taste or a secret beauty cream formula will remain an attractive feature as long as it is out of reach and cannot be manipulated (If consumers are ever allowed to manipulate them then what would be attractive to consumers is tools and platforms available for them to alter and manipulate the product).

On the other hand, from an innovation perspective, what mass customization and open innovation among other technologies create is a state of continuous innovation and development, a situation that is too hard and too costly to imitate otherwise. Once again, if the product core is continuously innovated (as in open innovation) or augmented (as in mass customization) by the help of the user, the company will focus many of its resources on the innovation of its platform (which can also be open sourced at certain levels) and on its role as a process, a service provider, and facilitator. This does not necessarily mean that the company is totally abdicating its full responsibilities in the development process but surely sharing many of them.

To some people, this idea of a brand with a score of distinctive features rather than one or a few common distinctive features might seem like a contradiction to what a brand is all about. Yet in reality, the idea of having just one key benefit is neither a reflection of how products are invented nor really a reflection of how people choose, buy, and appreciate them. It is mainly a requirement of a certain type of "hard sell" advertising. This has been the way things worked in a mass-produced environment in order to differentiate among similar products, which is obviously irrelevant in the new model. As a result, marketing and advertising strategies need to change in a way that is more responsive and accommodating to the new environment and the nature of the newly created satellite-brands. Under MC, a brand is inherently differentiated, and the sum of distinctive features created by the series of satellite brands do not qualify for the current massproduced, hard sell advertising strategies and marketing campaigns. There is a need for a more direct, personal, and focused approach to communication and marketing messaging, which some of the new alternatives offered by new technologies such as Internet seems to support (such as direct marketing and behavior targeting which monitors internet shoppers choices and preferences).

\section{Rethinking the branding process}

The most dramatic implication of these changes remains the redefinition of the relationship between the producer and the customer, as well as the role of each in this new relationship. The new relationship is that of a "partnership." This partnership changes the dynamics of brand development, as the role of the producer shifts from a product maker to mainly a service provider as we just mentioned. Within this framework, the promise of consistency is an element of service, image is a function of technical innovation, and the relationship is that of sharing rather than dictating. This by all measures is a major paradigm shift.

\section{Redefining the brand}

Based on what we have discussed, it seems inevitable that we consider revisiting the brand definition under the new environment to interpret these new relationships and influences. One interpretation that would focus on the effect of mass customization as a new and rising trend is:

A brand in a mass-customized environment is an experiential proposal manifested in unique product variations within a web of satellite micro brands created through a partnership between the manufacturer and the users, whereby the manufacturer establishes a brand identity, product base, and the platform and technology necessary for the user to co-design and define the desired variations. A mass-customized brand is inherently competitive, innovative, and pre-positioned with the goal of an economic return to its producer and a higher level of emotional and experiential value to its end user.

By comparing this definition to our initial one we notice a couple of important observations:

a. While the nature and structure of the brand may differ under the new model, its goal for financial return and generation of value remains the same.

b. The role branding plays in minimizing our purchase risks would seem to decline with the increase of user input. This may impose few threats as the customer gets more confident and ready to switch and try other alternatives. While that may indicate a lower propensity to brand loyalty, it really means that brands need to work harder and use innovative tools to maintain the customer. Keeping up with technological advances that facilitate the customization process and offering more and easier options can be one way to do this. Loyalty remains all about service.

c. Our initial definition implies that effective positioning is only achieved by some brands, especially the successful ones, while under the new model, every satellite brand is inherently pre-positioned most importantly by the end user himself. On the other hand, the mother brand is positioned more on the basis of experiential and emotional grounds than on a product one.

d. Many of the signs attributed to successful brands in the first definition such as innovation, consistency, and positive image are inherent in the new satellite brands and are most often influenced by the end user himself, which can lead to a higher (and probably unprecedented) level of satisfaction.

e. Finally, under the new model, the brand is in a continuous state of innovation that is, for the most part, relevant and with less financial burdens. A situation that is harder and much costly to emulate under the classic model of branding. ${ }^{1-25}$

\section{Conclusion}

It is safe to say that new technologies and models have a direct impact on the branding process in a manner that calls for a serious attempt to re-evaluate the process and the conventional "old-school" 
concepts associated with it. Our analysis demonstrates that these innovations create new principles for communicating and interacting with the customer, a stronger focus on experiential value, redefinition of the concepts of positioning and distinctiveness. This in return could strengthen the brand, improve its image, and increase customer loyalty. But above all, it would redefine the relationship between the brand, the company, and the end user.

Our attempt to understand the impact of these new technologies on the brand and to redefine it accordingly should not by any means be seen as the ultimate call on the new brand, it should instead be seen as a wakeup call and an invitation to further examine the new meaning and role of the brand in the twenty-first century.

\section{Acknowledgements}

None.

\section{Conflict of interest}

Author declares there is no conflict of interest in publishing the article.

\section{References}

1. Adamson Allen P. Brand Simple. 1st Edn. Palgrave Macmillan, New York, USA; 2006. p. 230.

2. Ahonen Tomi T, Alan Moore. Communities Dominate Brands. Future text Limited, London, UK; 2005. p. 274.

3. Brazeal Mickey. RFID: Improving the Customer Experience. Ithaca, USA: Paramount Publishing; 2009. p. 264.

4. Cherkoff J. What is open source marketing? 2005.

5. Cocoran Ian. The Art of Digital Branding. Allworth Press, New York, USA; 2007.

6. Davis Scott M. Brand Asset Management. Jossey-Bass, San Francisco, USA; 2000. p. 265.

7. Davis Scott S, Michael Dunn. Building the Brand-Driven Business. Jossey-Bass, San Francisco, USA; 2002. p. 320.

8. Diehl Gregory. Brand Identity Breakthrough: How to Craft Your Company's Unique Story to Make Your Products Irresistible. Identity Publications; 2016.
9. Gabor Deb. Branding is Sex: Get Your Customers Laid and Sell the Hell out Anything. Lioncrest Publishing; 2016. p. 202.

10. Canadian Marketing Association. Canadian Marketing Blog. CMA, Canada; 2017.

11. Hameide Kaled. Fashion Branding Unraveled. New York: Fairchild Publications; 2010. p. 290.

12. Hart Susannah, John Murphy. Brands: The New Wealth Creators. New York University, New York, USA; 1998.

13. Holt Douglas B. Brands and Branding. Boston: Harvard Business School Publishing; 2003.

14. Grant J. The Brand Innovation Manifesto. John Wiley \& Sons, West Sussex, England, UK; 2006. p. 330.

15. Kapferer Jean-Noel. Re-Inventing the Brand. Kogan Page Limited, London, UK; 2001. p. 240.

16. Kull Hans. Mass Customization: Opportunities, Methods, and Challenges for Manufacturers. 1st edn. Apress, New York, USA; 2015. p. 148.

17. Mathieson Rick. Branding Unbound. Amacom, New York, USA; 2005.

18. Meeker Heather. Open (Source) for Business: A Practical Guide to Open Source Software Licensing. North Charleston: Create Space Independent Publishing Platform; 2015. p. 344.

19. Meyers Herbert, Richard Gerstman.Branding@The Digital Age. Palgrave, New York, USA; 2001. p. 178.

20. Miller Jeremy. Sticky Branding: 12.5 Principles to Stand Out, Attract Customers, and Grow an Incredible Brand. Dundurn Press, Toronto, Canada; 2015.

21. Youngme E Moon. Rethinking Positioning. Boston: Harvard Business School Publishing; 2006. p. 34.

22. Nadeau Raymond A. Living Brands. New York: McGraw-Hill; 2007. p. 302.

23. Piller F, Moeslein K, Stoko C. Does mass customization pay? An economic approach to evaluate customer integration. Production Planning \& Control. 2004;15(4):435-444.

24. Schaefer Wolfgang, Kuehlwein JP. Rethinking Prestige Branding: Secrets of the Ueber-Brands. Kogan Page, London, UK; 2015. p. 272.

25. Zyman Sergio. The End Of Marketing As We Know It. New York: Harper Collins Publishers; 1999. p. 246. 